\title{
Trends in the Utilization of Total Wrist Arthroplasty versus Wrist Fusion for Treatment of Advanced Wrist Arthritis
}

\author{
Eitan Melamed, $\mathrm{MD}^{1}$ \\ Bryan Marascalchi, MD² \\ John T. Capo, $\mathrm{MD}^{1}$
${ }^{1}$ Division of Hand Surgery, NYU Hospital for Joint Diseases, New York, New York
2 Division of Spinal Surgery, Department of Orthopaedic Surgery, NYU Medical Center Hospital for Joint Diseases, New York, New York
${ }^{3}$ Division of Hand Surgery, Department of Orthopedics, Mayo Clinic, Rochester, Minnesota

Richard M. Hinds, $\mathrm{MD}^{1}$

Marco Rizzo, MD 3

\begin{abstract}
Address for correspondence Eitan Melamed, MD, Division of Hand Surgery. NYU Hospital for Joint Diseases, $301 \mathrm{E} 17$ th St., New York, NY (e-mail: Eitanme2000@yahoo.com).
\end{abstract}

J Wrist Surg 2016;5:211-216.

\begin{abstract}
Background Total wrist arthroplasty (TWA) provides the requisite range of motion to accomplish activities of daily living, especially for low-demand patients with bilateral wrist arthritis. However, there are no large epidemiologic studies to evaluate nationwide trends of TWA and wrist fusion (WF).

Questions/Purposes To analyze data collected from the National Inpatient Sample (NIS) to compare utilization, demographic, and outcome data among patients undergoing TWA versus total WF. We hypothesized that utilization rates of TWA have significantly increased over the prior decade in the United States.

Methods NIS data from 2001 to 2010 were reviewed. Procedures were identified by ICD-9-CM codes 81.73 (TWA) and 81.25 (WF). Utilization rates, primary treatment diagnoses, patient demographic and medical comorbidity data, and procedure costs were compared between TWA and WF.

Results There was a decrease in the number of procedures per year for TWA, while the number of WF remained relatively unchanged. There was, however, a transient increase in the frequency of TWA procedures performed from 2005 to 2008, following a decline in 2005. Patients with traumatic arthritis were more likely to receive WF. Rheumatoid patients were more likely to receive TWA. Patients receiving TWA tended to be older, female, be insured by Medicare, have a greater comorbidity burden, and have

Keywords

- arthritis

- National Inpatient Sample

- total wrist arthroplasty

- wrist fusion rheumatologic disease.

Conclusion WF was performed nearly four times more frequently than TWA. A trend was demonstrated toward a decreasing number of TWA being implanted, and in patients with more underlying comorbidities. TWA was associated with a higher hospitalization charge, which may be expected given the higher implant costs associated with arthroplasty.

Level of Evidence Level II, prognostic study.
\end{abstract}

received

October 8, 2015

accepted

January 4, 2016

published online

February 9, 2016
Copyright ( $\odot 2016$ by Thieme Medical Publishers, Inc., 333 Seventh Avenue, New York, NY 10001, USA. Tel: +1(212) 584-4662.
DOI http://dx.doi.org/ 10.1055/s-0036-1571841. ISSN 2163-3916. 
Wrist arthritis is commonly thought of as a relatively rare problem. ${ }^{1,2}$ In the early stages, symptomatic wrist arthritis can be managed nonoperatively using topical and oral medications, splinting, and activity modification. Less invasive, motion-preserving operative techniques including limited wrist fusion (WF), wrist denervation, and arthroscopic debridement may provide temporary relief and preservation of motion in cases unresponsive to conservative treatment. The two major options for the definitive treatment of recalcitrant, advanced wrist arthritis include total wrist arthroplasty (TWA) and total WF. ${ }^{3-6}$ Although WF is still considered by many to be the treatment of choice for management of advanced pancarpal wrist arthritis, patients frequently report limitations following fusion, involving activities such as perineal care, screwdriver use, button fastening, hair combing, and jar opening. ${ }^{6-8}$ Therefore, TWA has emerged as an alternative that appears to provide the requisite range of motion to accomplish activities of daily living, especially for low-demand patients with bilateral pancarpal arthritis. ${ }^{9}$ However, there are no large epidemiologic studies to evaluate nationwide trends of TWA and WF.

The purpose of this study was to analyze data collection from the National Inpatient Sample (NIS) to identify demographic and hospital data, incidence of comorbidities, and procedure-related complications associated with WF versus TWA. As a result of improved quality and design of implants, we hypothesized that the frequency of TWA performed has significantly increased over the prior decade in the United States.

\section{Materials and Methods}

We reviewed temporal trends in the utilization of TWA and WF from 2001 through 2010 using the NIS. The NIS database is the largest all-payer database of hospital discharges in the United States. ${ }^{10}$ The database was developed as a part of the Healthcare Cost and Utilization Project, which is sponsored by the Agency for Healthcare Research and Quality, which falls under the Department of Health and Human Services of the U.S. government. The database consists of approximately $20 \%$ stratified sample of U.S. hospitals. Because data in this study were de-identified, this study was exempt from review by the institutional review board. It does not contain clinical and radiographic data.

\section{Inclusion Criteria}

To identify the patients, we used the ninth revision of the International Classification of Diseases, clinical modification (ICD-9-CM). The database was searched for procedure codes of 81.73 indicating TWA and 81.25 indicating WF. Patients coded for both procedures were excluded. Individual comorbidity data were assigned using the Agency for Healthcare Research and Quality comorbidity variables, based on ICD-9CM codes. ${ }^{11}$ Comorbidities were identified using the following ICD-9 codes: hypertension (401, 402, 403, 404, 405), diabetes mellitus (250), anemia (285), liver disease (571.8), congestive heart failure (428), hypothyroidism (244.9), chronic pulmonary disease (490-492), coagulopathy (286), electrolyte imbalance (276), obesity (278), renal disease (582, $585,403)$, and metastatic and nonmetastatic cancer (199.1). Overall comorbidity burden was calculated using the Deyo comorbidity index, an adaptation of the Charlson comorbidity index. ${ }^{12}$ Both length of stay (LOS) and complication rates and cost were compared between the two treatment groups. The primary diagnoses listed in the database for the procedures evaluated were assessed by ICD codes 715.X3 and 715 . X4 for osteoarthritis (OA), 716.9 for unspecified arthropathy, 716.1 for traumatic arthropathy, 733.81 and 733.82 for malunion and nonunion, and 714.0 for rheumatoid arthritis (RA).

\section{Statistical Analysis}

Statistical analysis was performed using $\mathrm{R}$ foundation for statistical computing, (Vienna, Austria). Differences in patient and hospital characteristics were determined using chisquare tests for categorical variables and $t$-test for continuous variables. Linear regression modeling was used to determine the association between procedures and year. All data undergoing statistical analysis were original data from the database and not based upon extrapolated values.

\section{Results}

There were 199 TWA and 738 WF cases found in the NIS database during this 10 -year period. This was extrapolated to a national 10-year incidence corresponding to $\sim 995$ TWA cases and 3,960 WF procedures nationwide. The calculated nationwide numbers of WF and TWA in each year are shown in -Fig. 1. Over the 10-year duration reviewed, there was a decrease in the frequency (number of procedures per year) for TWA $(p=0.04)$, while the number of WF remained relatively constant $(p=0.13)$. There was, however, a transient increase in the frequency of TWA procedures observed from 2005 to 2008, following a decline in 2005, where the percentage of TWA increased from $10 \%$ in 2005 to $20 \%$ in 2006 and 2007 , and $24 \%$ in 2008 (percentage of total procedures). Finally, in 2010, there was a drop in both procedures (-Fig. 1).

\section{Demographic Characteristics}

Mean age of the patients who underwent TWA was 58.3 years versus 52 years in patients who underwent WF $(p<0.001)$. - Fig. 2 shows a comparison of mean age of patients in both groups during the study period. Of note, the mean age of the patients undergoing WF increased from 50 to 54 years $(p<0.05)$, while the age of TWA patients was unchanged. Of patients, $73 \%$ were female in the TWA group, as compared with $43 \%$ of patients in the WF group $(p<0.001)$. Analysis of ethnicity revealed that whites comprised $82 \%$ of the TWA and $77 \%$ of all fusions, African Americans $9 \%$ of the TWA and $12 \%$ of all fusions, and Hispanics $7 \%$ of the TWA and $8 \%$ of all fusions. The rate of whites receiving TWA was not significantly different than that of nonwhites. According to the 2010 census data, the racial categories in the United States include $72.4 \%$ whites, 12.6\% African Americans, 16.4\% Hispanics, and 4.8\% Asian. $^{13}$ 


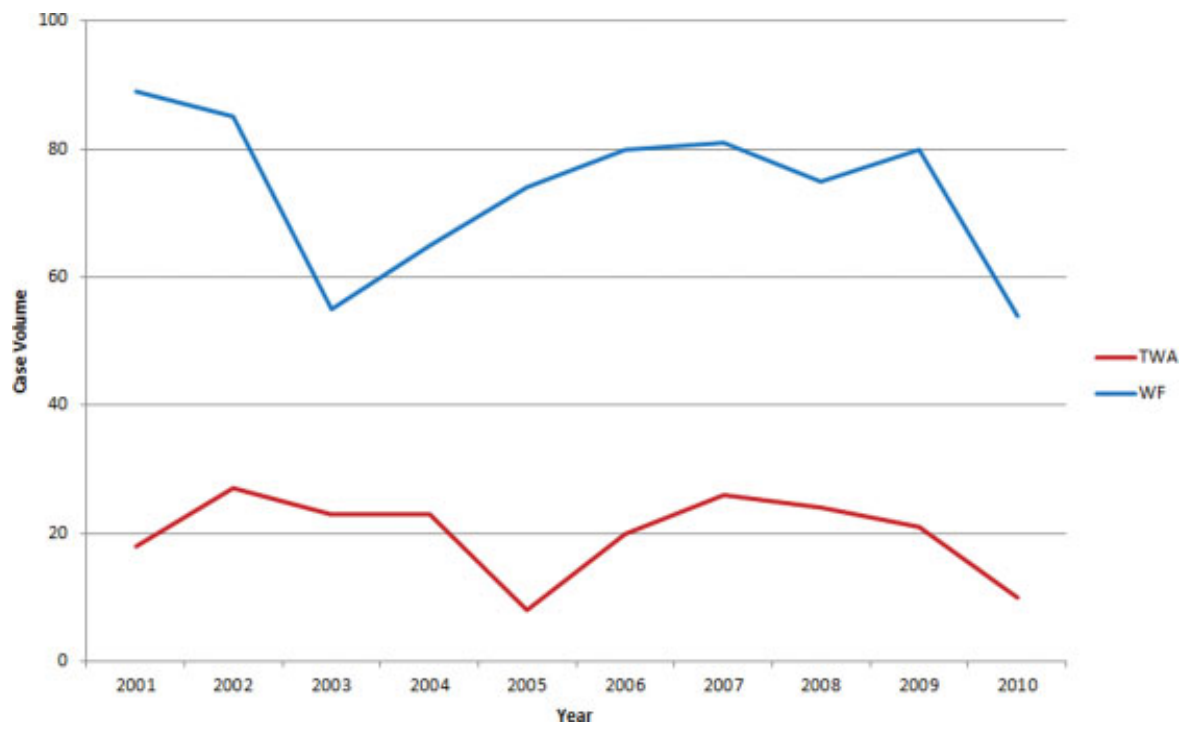

Fig. 1 National trends of volume of total wrist arthroplasty and total wrist fusion from 2001 to 2010.

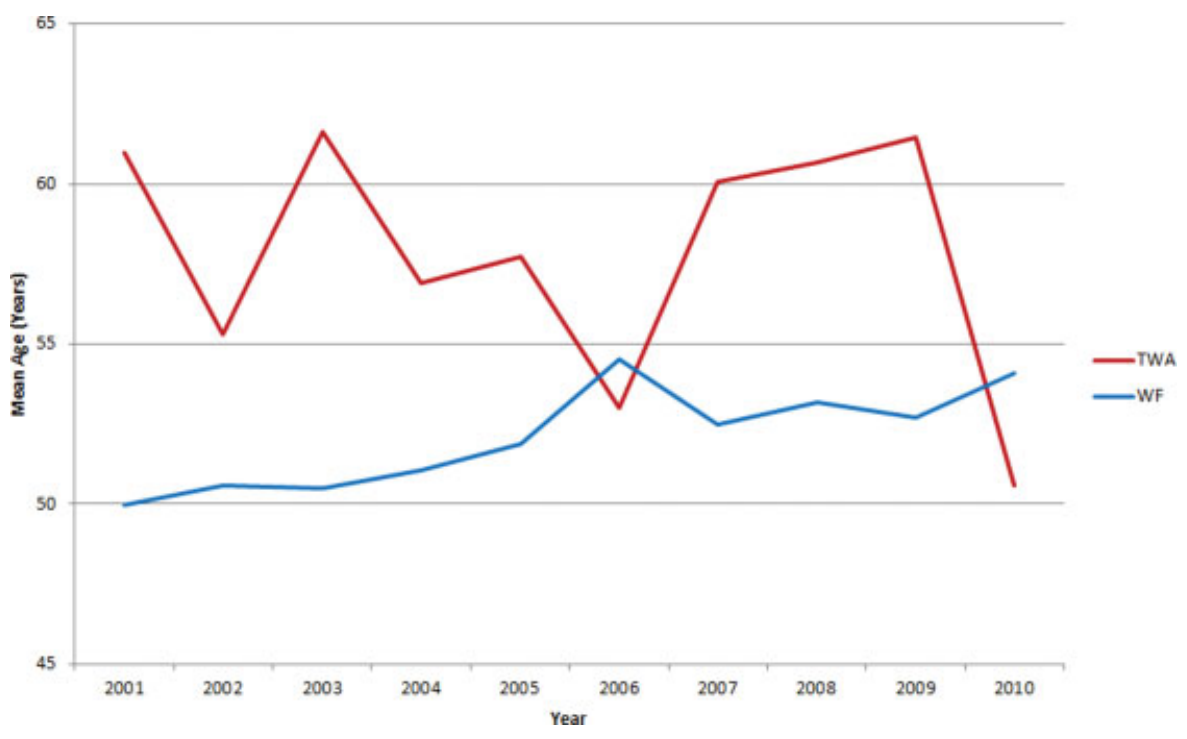

Fig. 2 Comparison of mean age of patients in total wrist arthroplasty and total wrist fusion groups.

\section{Comorbidities}

Overall comorbidity burden calculated by Deyo comorbidity index was greater for patient undergoing TWA (TWA: 0.97; WF: $0.54 ; p<0.001)$.

\section{Underling Diagnoses}

Based on the available coding system, RA was the most common underlying diagnosis for patients receiving TWA (51\% of the TWA cases), followed by OA (34\%) and unspecified arthropathy (6\%). In the WF group, OA was the most common underlying diagnosis (33\% of cases), followed by RA (16\%), traumatic arthropathy (12\%), and malunion (12\%). Statistical analysis showed that patients with malunion or traumatic arthritis were more likely to receive WF by percentage (malunion: 12 vs. $3 \%, p<0.001$; traumatic: 12 vs. $3 \%, p<0.001$ ). Patients with RA were more likely to receive TWA (RA: 51 vs.
$16 \%, p<0.001)$. The extrapolated total number of patients who underwent WF in each diagnostic group separately, and overall, outnumbered those who underwent TWA (995 TWA vs. 3,960 WF).

\section{Length of Stay and Cost}

Average LOS was a little over 2 days for both TWA and WF. Linear regression modeling showed that for WF, LOS increased from 1.7 in 2001 to 2.9 in 2010, but this trend did not reach statistical significance $(p=0.1$; - Fig. 3$)$. As seen in -Fig. 4, the cost of both procedures has risen over the last 10 years, with TWA being generally more expensive than WF. TWA was associated with a higher hospitalization charge $(\$ 34,055$ vs. $\$ 27,079, p<0.01)$, although both TWA and WF underwent a steady increase in hospitalization charge (-Fig. 4). 


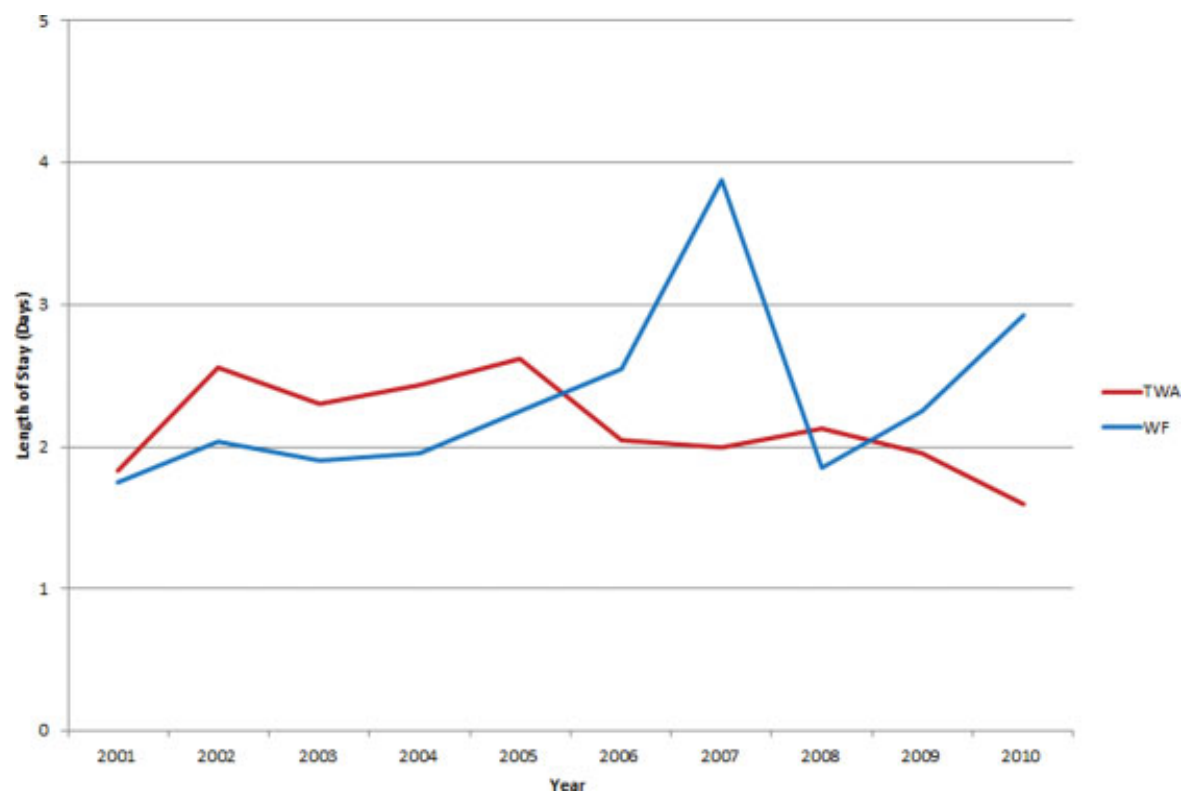

Fig. 3 Trends of length of stay for total wrist arthroplasty and wrist fusion from 2001 to 2010.

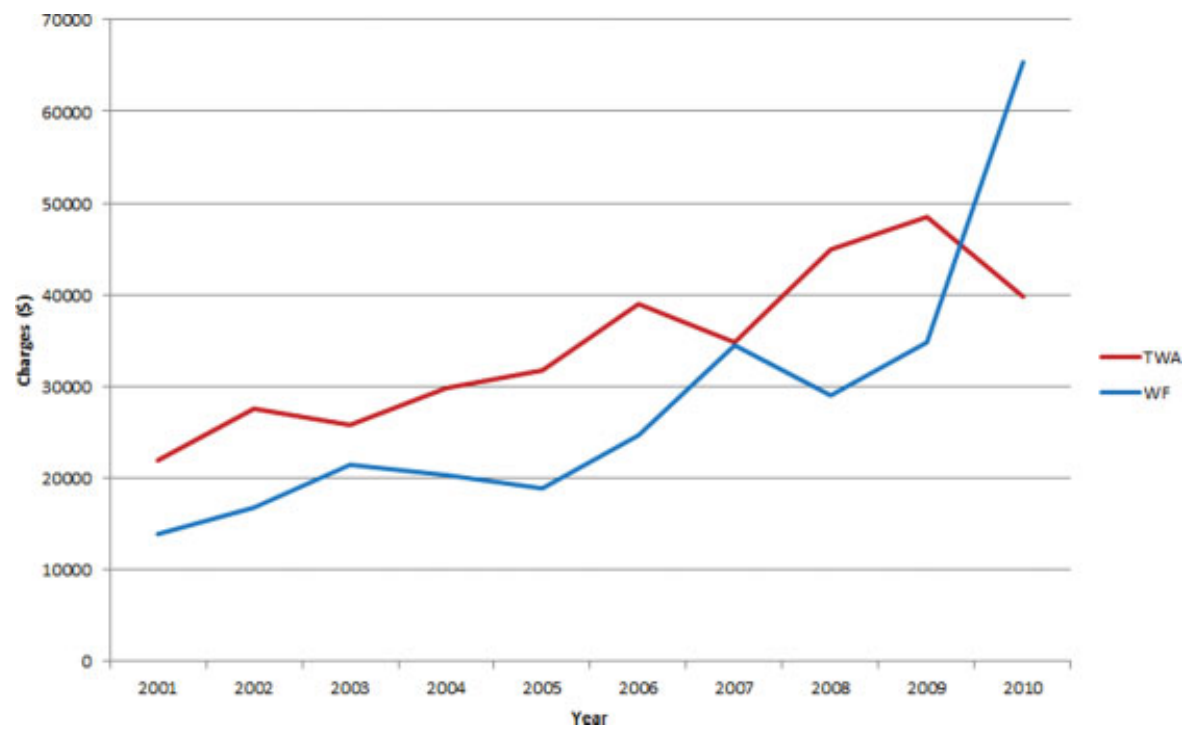

Fig. 4 Cost of hospitalization for TWA and WF.

\section{Complications}

Total complication rate was $10 \%$ for $\mathrm{WF}$ and $7 \%$ for TWA $(p=0.0033)$. However, these complications were not stratified as being either major or minor. Patients receiving WF had a higher percentage of perioperative device-related complications ( 6 vs. $3 \%, p<0.001)$ and respiratory complications (0.54 vs. $0 \%, p<0.05)$.

\section{Discussion}

WF and TWA are two common procedures for treatment of severe end-stage wrist arthritis nonresponsive to conservative management. Although WF is often still considered the treatment of choice for the management of pancarpal arthritis, some fourth-generation TWA implants have shown good midterm survival with low failure rates. ${ }^{14-18}$ Yet, poor results have also been demonstrated. ${ }^{19,20}$ However, there has been no large epidemiologic study to evaluate nationwide trends of WF and TWA. To the best of our knowledge, the present study is the first report of national utilization of TWA and WF for treatment of advanced wrist arthritis.

Despite improvement in design and kinematics of newer generation TWA systems, and the potential for preservation of motion, WF was still performed about four times more frequently. Close analysis of the overall trend showed an initial surge in the early 2000s, followed by a decline in 2005 . We assume that this decrease may be partly explained by the withdrawal of the Biax prosthesis (Depuy Orthopaedics) in 2004, which was one of the few FDA-approved wrist implants at the time. The Biax TWA was introduced in 1983 and its production was discontinued in 2004 without having developed a follow-up model. ${ }^{21}$ Overall, the 10 -year trend was that 
of decreasing numbers of TWA being implanted. This may be related to the advent of biologic disease-modifying drugs for RA, which have decreased the rate of progression to painful, end-stage arthritis. ${ }^{22}$ As these medications effectively prevent disease progression, fewer patients are expected to require reconstruction of the radiocarpal joint compared with previous decades. In addition, there are several alternative reconstruction options for the rheumatoid wrist that can potentially decrease the number of TWA procedures performed. However, limited WF are appropriate in patients with early collapse patterns of the wrist and in those with destruction limited to only a portion of the radiocarpal joints. ${ }^{23}$ Another theory is that since training to perform TWA is not standard in most hand fellowship programs, surgeons will avoid procedures that are outside their training or comfort level. A final explanation may be a trend toward TWA procedures being done on an outpatient basis, thus decreasing the number of procedures being represented in the NIS database, which only accounts for inpatient surgery. Indeed, younger and healthier non-RA patients are more likely to be treated on an outpatient basis.

We are not sure as to why numbers of both procedures dropped in 2010. Since the data were retrieved in mid-2013, we would expect that all 2010 cases had been reported. A possible explanation is more procedures done in an outpatient setting, and thus a lesser number of procedures is represented, but that still would not explain the sudden drop.

Based on this study, the most common joint disorder resulting in TWA was RA (51\%), followed by OA and unspecified arthropathy. The most common diagnosis resulting in WF was OA, followed by RA, traumatic arthropathy, and malunion. The present study indicates that RA patients are more likely to receive TWA than WF. Traditionally, the ideal candidates for TWA have been RA patients who comparatively lead a less active lifestyle. In a recent systemic review summarizing the results of eight articles published in the past 5 years, RA was the indication for surgery in $42 \%$ of the cases that provided the primary patient diagnosis. ${ }^{20}$ Furthermore, several other studies have described RA as an indication for TWA but have found variable outcomes. ${ }^{19,24}$ Additionally, two other studies found no difference in revision rates between the different diagnostic etiologies. ${ }^{16,25}$ These studies include data on the use of fourth-generation TWA prostheses. Our cohort includes the years from 2001 to 2010, and although NIS does not indicate the generation of TWA prosthesis used, we speculate that before 2010, use of these newer implants was more limited. Likewise, with the advent of new implant technology, TWA is increasingly offered as a treatment option to patients with posttraumatic arthritis and OA if they are able to maintain certain activity restrictions. ${ }^{26}$ Currently, fourth-generation implants are available in the market. Fourth-generation designs have been proposed in an attempt to solve the complications with previous models, mainly loosening of the distal component. Fourth-generation implants are mainly uncemented with porous titanium surfaces to allow for osseous integration. Although fourth-generation implants have shown promise, additional long-term outcome studies as well as prospective randomized trials are needed to compare TWA with WF in the management of pancarpal arthritis.

This study has several limitations that need to be recognized. The NIS database accounts for inpatient surgery only, while today many patients undergo both of these procedures on an outpatient basis. These patients were not accounted for in this database review, potentially underestimating the number of procedures performed. In addition, morbidities occurring after discharge are not captured. Thus, the findings of this study are limited to the acute perioperative period, and may underestimate the actual complication rates. Another limitation is that we were only able to report the incidence (new cases in a given time period) of TWA or WF. The prevalence (all existing cases) of TWA or WF remains unknown. While many of the results of this study are expected, namely, TWA is done more often in RA patients and that there is a very low rate of significant perioperative medical complications, it is still important to quantify this for physicians and patients. Also, the data are limited by the coding done by the hospitals, and may have had inaccuracies.

In conclusion, our analysis of this national inpatient database registry from 2001 to 2010 demonstrates that the rate of WF remains essentially constant, while the rate of TWA is on a gentle downward trend. This applies for patients undergoing inpatients surgery only, and cannot be generalized to all cases. In addition, the cost of both procedures has risen over the last 10 years, with TWA being generally more expensive than WF.

Ethical Review Committee

The study does not involve patient data and did not require ethical committee as the data used were de-identified.

Note

The study was performed entirely at NYU Hospital for Joint Diseases, New York.

Conflict of Interest

None.

\section{References}

1 Buckwalter JA, Saltzman C, Brown T. The impact of osteoarthritis: implications for research. Clin Orthop Relat Res 2004(427, Suppl): S6-S15

2 Watson HK, Ballet FL. The SLAC wrist: scapholunate advanced collapse pattern of degenerative arthritis. J Hand Surg Am 1984; 9(3):358-365

3 Graham TJ. Total wrist arthrodesis and total wrist arthroplasty. In: Chhabra AB, Issacs J, eds. Arthritis and Arthroplasty: The Hand, Wrist and Elbow. 1st ed. Philadelphia, PA: Saunders; 2009:90-105

4 Nydick JA, Watt JF, Garcia MJ, Williams BD, Hess AV. Clinical outcomes of arthrodesis and arthroplasty for the treatment of posttraumatic wrist arthritis. J Hand Surg Am 2013;38(5): 899-903 
5 Cavaliere CM, Chung KC. A systematic review of total wrist arthroplasty compared with total wrist arthrodesis for rheumatoid arthritis. Plast Reconstr Surg 2008;122(3):813-825

6 Murphy DM, Khoury JG, Imbriglia JE, Adams BD. Comparison of arthroplasty and arthrodesis for the rheumatoid wrist. J Hand Surg Am 2003;28(4):570-576

7 Anderson MC, Adams BD. Total wrist arthroplasty. Hand Clin 2005; 21(4):621-630

8 Graham TJ, Harman TW, Higgins JP, et al. Rheumatoid wrist arthrodesis using plate fixation. Atlas Hand Clin 2005;10:289-305

9 Franko OI, Zurakowski D, Day CS. Functional disability of the wrist: direct correlation with decreased wrist motion. J Hand Surg Am 2008;33(4):485-492

10 Agency for Healthcare Research and Quality. HCUP NIS database documentation. Avalable at: http://www.hcup-us.ahrq.gov/db/ nation/nis/nisdbdocumentation.jsp. Accessed July 18, 2014

11 Agency for Healthcare Research and Quality. Healthcare Cost and Utilization Project (HCUP). HCUP comorbidity software. Available at: http://www.hcup-us.ahrq.gov/toolssoftware/comorbidity/. Accessed July 18, 2014

12 Charlson ME, Pompei P, Ales KL, MacKenzie CR. A new method of classifying prognostic comorbidity in longitudinal studies: development and validation. J Chronic Dis 1987;40(5):373-383

13 US Census Bureau. 2010 census. Available at: http://www.census. gov/population/race/data/cen2010.html. Accessed July 24, 2014

14 Morapudi SP, Marlow WJ, Withers D, Ralte P, Gabr A, Waseem M. Total wrist arthroplasty using the Universal 2 prosthesis. J Orthop Surg (Hong Kong) 2012;20(3):365-368

15 Herzberg G. Prospective study of a new total wrist arthroplasty: short term results. Chir Main 2011;30(1):20-25

16 Herzberg G, Boeckstyns M, Sorensen AI, et al. "Remotion” total wrist arthroplasty: preliminary results of a prospective international multicenter study of 215 cases. J Wrist Surg 2012;1(1):17-22
17 Ferreres A, Lluch A, Del Valle M. Universal total wrist arthroplasty: midterm follow-up study. J Hand Surg Am 2011;36(6):967-973

18 Boeckstyns ME, Herzberg G, Merser S. Favorable results after total wrist arthroplasty: 65 wrists in 60 patients followed for 5-9 years. Acta Orthop 2013;84(4):415-419

19 Harlingen Dv, Heesterbeek PJ, J de Vos M, High rate of complications and radiographic loosening of the biaxial total wrist arthroplasty in rheumatoid arthritis: 32 wrists followed for 6 (5-8) years. Acta Orthop 2011;82(6):721-726

20 Ward CM, Kuhl T, Adams BD. Five to ten-year outcomes of the Universal total wrist arthroplasty in patients with rheumatoid arthritis. J Bone Joint Surg Am 2011;93(10):914-919

21 Kretschmer F, Fansa H. BIAX total wrist arthroplasty: management and results after 42 patients [in German]. Handchir Mikrochir Plast Chir 2007;39(4):238-248

22 Nikiphorou E, Carpenter L, Morris S, et al. Hand and foot surgery rates in rheumatoid arthritis have declined from 1986 to 2011, but large-joint replacement rates remain unchanged: results from two UK inception cohorts. Arthritis Rheumatol 2014;66(5): 1081-1089

23 Feldon P, Terrono AL, Nalebuff EA, Millender LH. Rheumatoid arthritis and other connective tissue diseases. In: Wolfe SW, Pederson WC, Hotchkiss RN, Kozin SH, eds. Green's Operative Hand Surgery. Philadelphia, PA: Churchill Livingstone; 2011: 1993-2065

24 Yeoh D, Tourret L. Total wrist arthroplasty: a systematic review of the evidence from the last 5 years. J Hand Surg Eur Vol 2015;40(5): 458-468

25 Krukhaug Y, Lie SA, Havelin LI, Furnes O, Hove LM. Results of 189 wrist replacements. A report from the Norwegian Arthroplasty Register. Acta Orthop 2011;82(4):405-409

26 Weiss AP, Kamal RN, Shultz P. Total wrist arthroplasty. J Am Acad Orthop Surg 2013;21(3):140-148 